\title{
International Society for Disease Surveillance Conference 2010: Enhancing the Synergy Between Research, Informatics, and Practice in Public Health
}

\author{
A Baer ${ }^{1}$, DR Olson ${ }^{2}$, and JS Brownstein ${ }^{3}$ \\ ${ }^{1}$ Public Health, Seattle \& King County, Seattle, WA, USA; ${ }^{2}$ International Society for Disease Surveillance, New York, NY, USA and ${ }^{3}$ Children's \\ Hospital Informatics Program at the Harvard-MIT Division of Health Sciences and Technology, Boston, MA, USA \\ E-mail: drolson@gmail.com
}

The International Society for Disease Surveillance (ISDS) was created to improve population health by advancing the field of disease surveillance. In support of this mission, ISDS provides educational and scientific forums where researchers, informaticists, public health practitioners, and healthcare providers can explore and address population health monitoring across institutional and professional boundaries.

As the scope of many journals tends to be focused on only a single discipline, advances in the field of population surveillance have been scattered across the scientific literature. In order to improve opportunities for its diverse membership to publish its work in a more cohesive way, ISDS has sought to create a partnership with a journal that will promote publication of cross-disciplinary approaches to population health monitoring. Also, acknowledging the severe financial pressures in the public health setting, ISDS sought a journal that was both free to publish in and openly accessible. ISDS is proud to announce its new partnership with the Emerging Health Threats Journal (EHTJ). As a first step towards this partnership, ISDS and EHTJ are collaborating to publish the 2010 ISDS Annual Conference abstracts.

The ISDS annual conference brings together researchers and practitioners in public health, epidemiology, health policy, biostatistics and mathematical modeling, informatics, computer science, and related fields focused on biosurveillance and emerging challenges to public health practice. The theme of the ninth annual conference, held on 1 and 2 December in Park City, Utah, was, 'Enhancing the Synergy Between Research, Informatics, and Practice in Public Health.' The conference was preceded by a workshop focused on disease surveillance standards, the interaction between disease surveillance and the International Health Regulations, and an introduction to syndromic surveillance. There were also several satellite meetings and user's groups focused on syndromic surveillance systems, global surveillance, and syndrome standardization.

The conference featured plenary, panel, and parallel sessions covering a wide range of topics addressing the following themes: Analytics/Research Methodologies, Public Health Surveillance/Practice, Applications of Methodologies to New Domains, Informatics Applications/Practice, and Informatics Architectures/Integration/Interoperability. A keynote address on bridging theory, technology, and practice in public health surveillance was given by Dr Samuel Groseclose, Acting Director of the Division of Healthcare Information, Public Health Surveillance Program Office (PHSPO), US Centers for Disease Control \& Prevention. A special call for proposals was announced to address the topic of data quality, which was featured as a panel presentation. Other plenary and panel presentations focused on the implications of meaningful use on research and practice, and the use of social networking tools for surveillance. A total of 186 abstracts were submitted to the conference; abstracts accepted for either an oral or poster presentation are published in this supplemental issue of the Journal. 